\title{
11. Deutscher Allergiekongress, Berlin
}

\section{Perlen aus dem DAK-Programm}

Dieses Jahr treffen sich Deutschlands Allergologen vom 30. September bis 1. Oktober in der Hauptstadt, um sich über neueste Forschungsergebnisse rund um die Allergologie auszutauschen. In der letzten Ausgabe haben wir Ihnen schon einige Highlights aus dem Programm vorgestellt. Heute setzen wir dies fort und möchten Ihnen noch weitere interessante Veranstaltungen, die Sie in Berlin erwarten, ans Herz legen.

\section{Urtikaria: Klartext zu Omalizumab}

- Gibt es noch Mysterien in der modernen Medizin? Therapeutische Durchbrüche bei ungeklärtem Mechanismus? Dramatische Effekte nach kurzer Zeit? Willkommen in der Welt der therapierefraktären chronischen Urtikaria bei Behandlung mit AntiIgE! Überzeugende Daten, internationale Zulassung in Rekordzeit und ein wohlbekanntes, sehr gutes Sicherheitsprofil: Die Erfolgsstory von Omalizumab hatte niemand geahnt. Erfahren Sie von Profis wie Anti-IgE in der Praxis oder Klinik einsetzt wird, welche Patienten in Frage kommen und was es zu berücksichtigen gilt. Diskutieren Sie mit, wann sich bei Kindern, Jugendlichen oder Erwachsenen ein Versuch lohnt und wie es dann weitergeht: Aufklä- rung, Monitoring, Verlaufskontrolle, Kalkulation der Therapiekosten oder einfach lebenslänglich (ein hässliches Wort). Verlassen Sie sich darauf: Die Moderatoren werden Klartext reden!

\section{AllergoActive: „Anti-IgE (Omalizumab) bei Urtikaria 2016: Wie geht's?"}

Donnerstag, 29. September 2016, 9.00-10.00 Uhr

\section{Aufklärung rund um die Anaphylaxie}

- Die Anaphylaxie - der allergische Mega-GAU - verdient echten Respekt und die

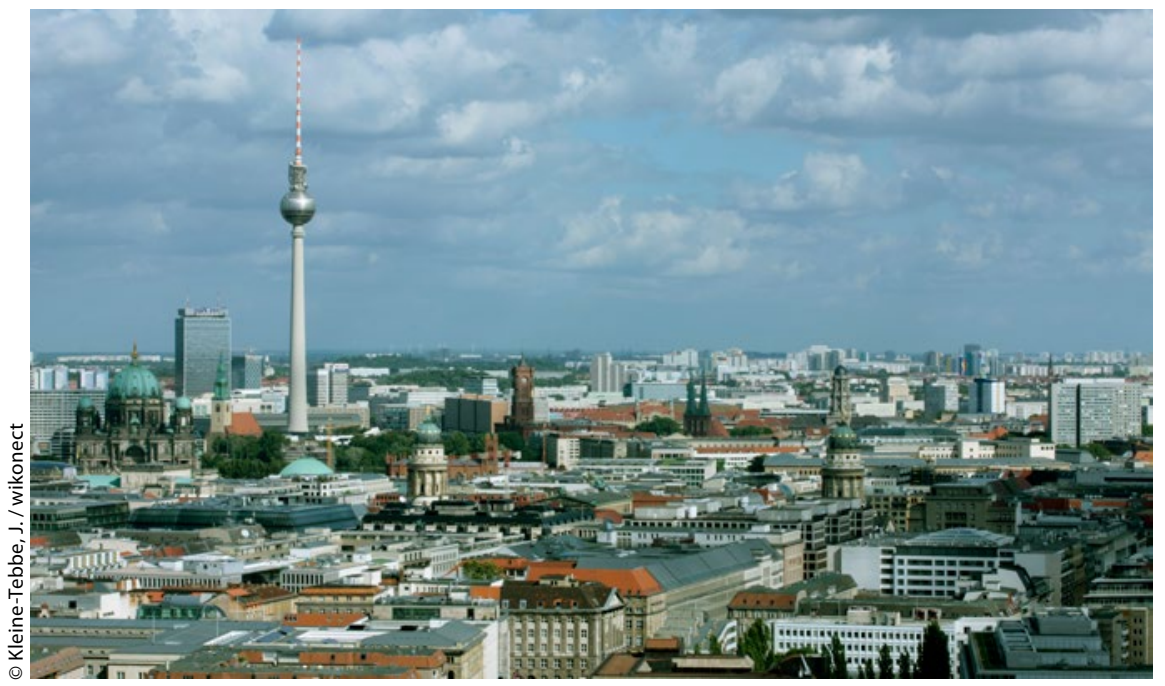

betroffenen Allergiker unsere größte Fürsorge. Ein Rezept mit Notfallmedikamenten nach gesicherter Diagnose ist definitiv nicht genug. Strukturierte Aufklärung, Beratung und Schulung sind hier gefragt. Profis erklären Ihnen wie das funktioniert: Wie werden Anaphylaxie und Risiken auf "gut deutsch" vermittelt? Welche Auslöser sind für wen relevant? Wie soll die Anwendung der Notfallmedikamente, vor allem des Adrenalin-Autoinjektors geübt werden? Wie wird ein Notfallmanagement installiert und wer wird eingebunden? Familie, Kindergarten, Schule, Arbeitsplatz, Nachbarn bis zur Kirche im Dorf? Profitieren Sie von der praktischen Erfahrung der Anaphylaxie-Profis und bringen Sie bitte Ihre Fragen mit.

AllergoActive: „Anaphylaxie 2016: Schulung und Management bei Kindern und Erwachsenen"

Donnerstag, 29. September 2016, 10.30-11.30 Uhr

\section{Provokante Fortbildung}

— Provokationstests haben einen hohen Stellenwert für die Allergiediagnostik. Trotzdem werden sie zu selten durchgeführt - der unzureichenden Vergütung geschuldet. Dabei steuern sie objektive Fakten zur klinischen Relevanz bei, besonders wenn die Vorgeschichte unklar ist: "Ente oder Trente", "hip" oder "hop" - so können "stumme" Sensibilisierungen von „echten" Allergien unterschieden werden. Nasale Provokationen mit Allergenen las-

Im Herbst in die Hauptstadt? Der Deutsche Allergiekongress vom 30.09. bis 1.10. ist ein passender Anlass für Allergologen die Metropole zu besuchen. 